\title{
How the Dirac Sea Idea May Apply to a Spatially-Flat Universe Model (A Brief Review)
}

\author{
Eugene Terry Tatum \\ Bowling Green, KY, USA \\ Email: ett@twc.com
}

How to cite this paper: Tatum, E.T. (2019) How the Dirac Sea Idea May Apply to a Spatially-Flat Universe Model (A Brief Review). Journal of Modern Physics, 10, 974-979.

https://doi.org/10.4236/jmp.2019.108064

Received: June 17, 2019

Accepted: July 14, 2019

Published: July 17, 2019

Copyright $\odot 2019$ by author(s) and Scientific Research Publishing Inc. This work is licensed under the Creative Commons Attribution International License (CC BY 4.0).

http://creativecommons.org/licenses/by/4.0/

\begin{abstract}
The famous Dirac sea idea can be resurrected if one replaces the concept of positive and negative matter mass with positive and negative energy. Utilizing this concept, the perpetually spatially-flat matter-generating FSC model can be shown to be a realistic Milne "empty universe" model. Furthermore, this may be why $R_{h}=c t$ cosmological models like FSC show an excellent statistical fit with the accumulated data of the Supernova Cosmology Project.
\end{abstract}

\section{Keywords}

Dirac Sea, Dirac Equation, Flat Space Cosmology, Dark Energy, Dark Matter, Inflationary Cosmology, Supernova Cosmology Project, $R_{h}=c t$ Models

\section{Introduction and Background}

Mathematical physicist Paul Dirac is perhaps best known for the Dirac equation in its many forms [1]. Not only was he largely responsible for making quantum mechanics relativistic, but his "hole theory" (based upon his equation) suggested to him something about the nature of the cosmic vacuum. Dirac believed that his equation implied that the vacuum could be a many-body quantum state in which all of the negative energy eigenstates (holes) are occupied. In terms of electron eigenstates, for instance, Dirac pictured a "sea" of electrons occupying negative-energy electron eigenstates. Unfortunately, his "Dirac sea" idea was interpreted to imply holes of negative mass matter, which is believed to be impossible. The term "antimatter" is used not to imply negative mass matter, but rather gravitationally-attractive positive-energy matter of the same mass as its positive-energy partner, yet opposite in quantum spin and charge. The combined mass-energy of an electron and positron, for instance, sums to twice that of the electron alone, rather than summing to zero. Despite the initial apparent failure of the Dirac sea idea, the Dirac equation was correctly credited with pre- 
dicting oppositely-charged matter of equal positive mass, for which the term antimatter is restricted.

On a parallel track, there is the currently-favored theory that our universe may have started from a zero-energy state and undergone a brief $\left(10^{-32} \mathrm{~s}\right)$ period of "cosmic inflation" in which all matter and antimatter were created. Nevertheless, this nearly instantaneous matter-generating universe theory would appear to violate conservation of energy. This did not escape notice by its inventor, Alan Guth [2] [3]. Guth has sometimes referred to his theory as a "free lunch" idea. Other somewhat modified inflationary theories [4] [5] of a nearly instantaneous matter-generating universe have followed, although the problem of energy conservation violation appears to be inherent in all such theories [6].

Recently, there have been proposed several perpetually matter-generating universe theories [7]-[14], which smoothly expand, are not inflationary in nature, and do not appear to violate conservation of energy. They are also consistent with current observations of a spatially-flat universe. One of the most successful of these theories, in terms of predicting current observations, is the "Flat Space Cosmology" (FSC) model. By following its five basic assumptions, the heuristic FSC model perpetually maintains the Friedmann critical mass density for a spatially-flat universe $\left(\rho_{0}=\frac{3 H_{0}^{2}}{8 \pi G}\right)$ for any theoretical time of observation (o). Furthermore, the FSC model tightly correlates the current redshifted cosmic microwave background (CMB) temperature of $2.72548 \mathrm{~K}$ with a current predicted Hubble parameter value of $66.9 \mathrm{~km} \cdot \mathrm{s}^{-1} \cdot \mathrm{Mpc}^{-1}$. This Hubble parameter value fits within the tight constraints of the 2018 Planck Collaboration [15] and 2018 Dark Energy Survey [16] reports.

\section{Relevance of the Dirac Sea Idea to the FSC Model}

The relevance of the Dirac sea idea to the FSC model pertains to its perpetual matter generation and its perpetual spatial flatness. As detailed in several 2018 publications [11] [12] [13], a globally perpetually-flat spacetime implies that the cosmological model must always maintain equal amounts of positive and negative energy. Otherwise, the more dominant energy density component would contribute an observable curvature signifying either cosmic deceleration (positive curvature) or cosmic acceleration (negative curvature). As the excellent statistical fit between $R_{h}=c t$ cosmological models and observations of the Supernova Cosmology Project indicates, the expansion of our universe appears to be coasting at constant velocity [7] [17] [18] [19] [20]. All $R_{h}=c t$ models have this "coasting at constant velocity" feature. For specifics concerning the basic features of $R_{h}=c t$ models, the reader is encouraged to start with these references. FSC is one such $R_{h}=c t$ model.

The "Dirac sea" idea can be resurrected if one considers a dichotomy of positive and negative energy states popping into and out of existence within the vacuum. If one follows the convention that all units of matter mass-energy are 
"positive" energy, then one can consider the "holes" in the Dirac sea to be similarly-sized units of "negative" vacuum energy. Furthermore, since the FSC model uses such a sign convention, the negative energy holes in the FSC Dirac sea can now be understood to be units of dark energy exactly offset by the units of matter mass-energy produced in the FSC vacuum. By this perpetually ongoing process, the FSC model accumulates increasingly positive (matter) energy and increasingly negative dark energy of equal magnitude, always summing to zero total energy. In this way, a universe which begins in a zero-energy state maintains perpetual conservation of total (i.e., global) cosmic energy.

\section{Results: Evidence in Support of FSC and Dirac}

The "net zero energy" FSC model can now be contrasted with standard inflationary cosmology, which considers such models to be unrealistic "empty universe" models. The phrase "empty universe" has generally been applied in the past to Milne-type models containing no matter. However, the current $R_{h}=c t$ models contain matter and, as such, are considered to be more realistic than Milne's original conception. If one looks carefully at the following open source graph [21] published by the Supernova Cosmology Project (SPC) (Figure 1), one can see the excellent statistical fit of the "empty" universe line demarcating the boundary between accelerating and decelerating universal expansions.

This "empty" universe line falls exactly where the FSC and other $R_{h}=c t$ models fall. One can also readily see how it is that $R_{h}=c t$ universe models appear to show an excellent statistical fit with SCP observations to date. For additional proof, another open source graph [22] published by the SCP is provided (Figure 2).

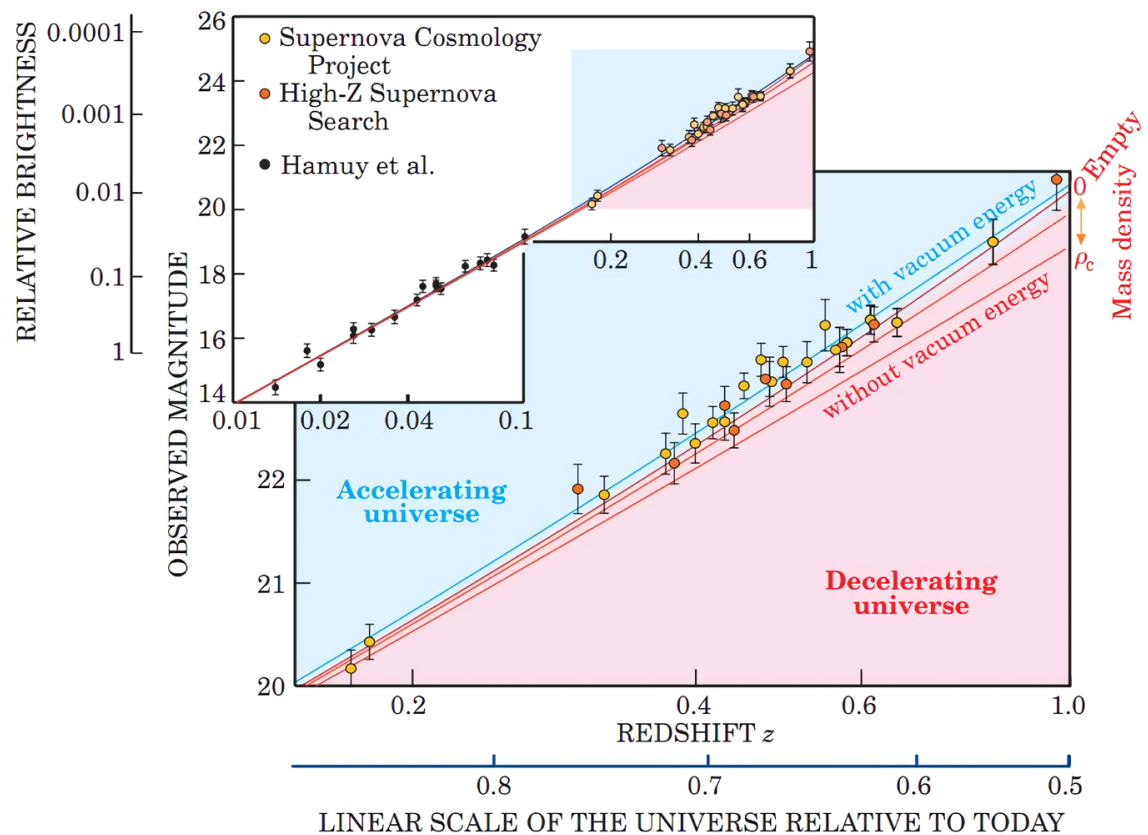

Figure 1. Observed magnitudes of type I a supernovae vs Redshift $z$. 


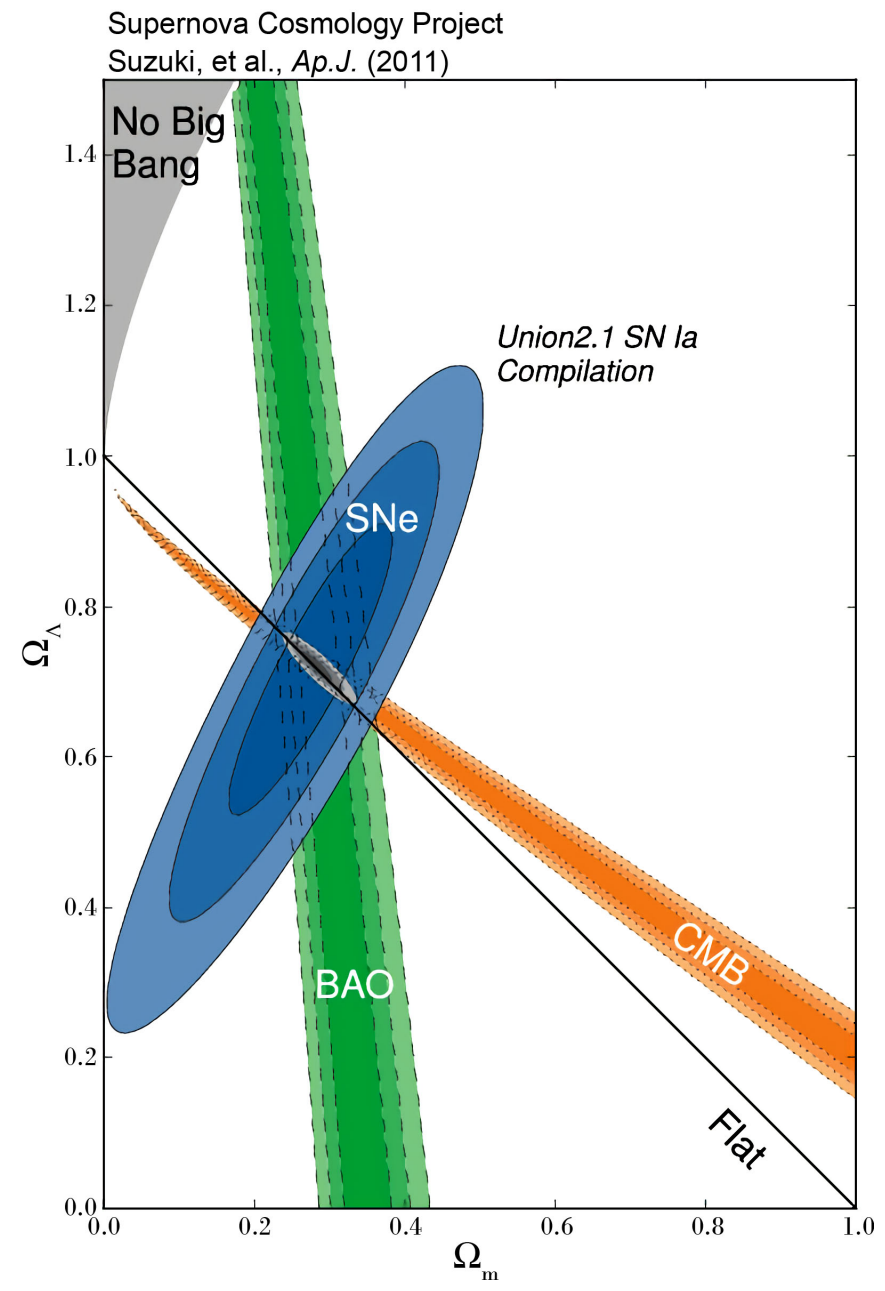

Figure 2. SCP supernovae, BAO and CMB data.

The "flat" line is where the perpetually spatially-flat FSC model falls. Once again, one can readily see that the FSC model shows an excellent statistical fit with the accumulated SCP data.

The significance of the use of the FSC model to resurrect the Dirac sea concept (at least in terms of opposite sign cosmic energies) is perhaps best seen in Figure 3. This graph is copied, in slightly modified form, from a 2018 FSC publication [23] incorporating the Bekenstein-Hawking entropy into the model in order to represent the cosmic clock as well as the "entropic arrow of time".

One can readily see that the magnitude of positive matter mass-energy (visible plus dark matter) of the FSC model scales in exactly the same way as the magnitude of negative dark energy scales. Thus, the "net zero energy" of the universe as a global object is always maintained. In this context, the "net zero energy" FSC model can be thought of as a realistic Milne-type "empty universe" model!

\section{Discussion and Summary}

This paper provides a brief look at Dirac's thought process concerning how the cosmic vacuum might behave if it follows his famous equation. The "Dirac sea" 


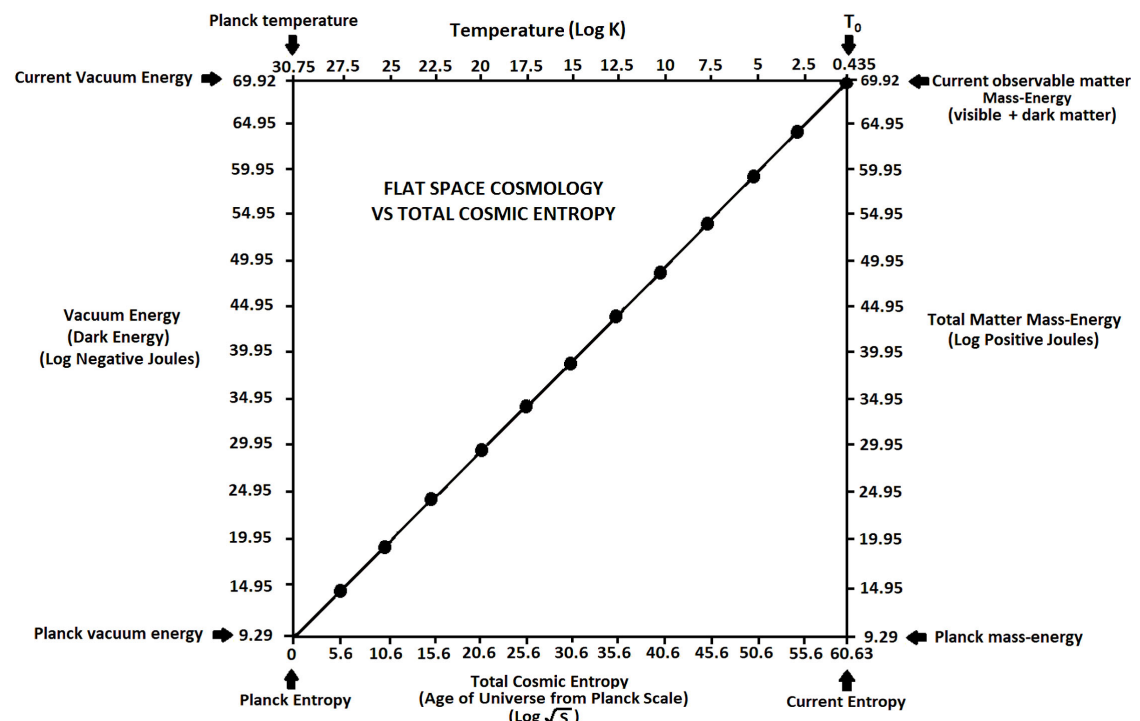

Figure 3. Flat space cosmology positive and negative energies vs cosmic time.

idea is resurrected in terms of a zero-point energy vacuum in which energy has positive (i.e., matter) and negative (i.e., dark energy) values always summing to zero (i.e., "net zero energy"). As it turns out, the FSC model, by its perpetual matter generation and its perpetual spacetime flatness, can be seen as a realistic Milne-type "empty universe" model. The genius of Paul Dirac and his equation can once again be readily seen when his "Dirac sea" idea for positive and negative matter (an impossibility) is resurrected in terms of positive and negative energy.

\section{Dedications and Acknowledgements}

This paper is dedicated to Stephen Hawking and Roger Penrose for their groundbreaking work on black holes and their possible application to cosmology. Dr. Tatum sincerely thanks U.V.S. Seshavatharam for his co-authorship of the seminal FSC papers and some of the more recent FSC publications. He also thanks Dr. Rudolph Schild of the Harvard Center for Astrophysics for his past support and encouragement.

\section{Conflicts of Interest}

The author declares no conflicts of interest regarding the publication of this paper.

\section{References}

[1] Wikipedia Contributors (2019) Dirac Equation. Wikipedia, the Free Encyclopedia. https://en.wikipedia.org/wiki/Dirac_equation

[2] Guth, A.H. (1981) Physical Review D, 23, 347. https://doi.org/10.1103/PhysRevD.23.347

[3] Guth, A.H. (1997) The Inflationary Universe. Basic Books, New York.

[4] Albrecht, A. and Steinhardt, P.J. (1982) Physical Review Letters, 48, 1220-1223. 
https://doi.org/10.1103/PhysRevLett.48.1220

[5] Linde, A.D. (1982) Physics Letters B, 108, 389-392.

https://doi.org/10.1016/0370-2693(82)91219-9

[6] Steinhardt, P.J. (2011) Scientific American, 304, 18-25. https://doi.org/10.1038/scientificamerican0411-36

[7] Melia, F. (2012) Astronomical Journal, 144, Article ID: 110.

[8] Tatum, E.T., Seshavatharam, U.V.S. and Lakshminarayana, S. (2015) International Journal of Astronomy and Astrophysics, 5, 116-124. https://doi.org/10.4236/ijaa.2015.52015

[9] Tatum, E.T., Seshavatharam, U.V.S. and Lakshminarayana, S. (2015) Journal of Applied Physical Science International, 4, 18-26.

[10] Tatum, E.T., Seshavatharam, U.V.S. and Lakshminarayana, S. (2015) Frontiers of Astronomy, Astrophysics and Cosmology, 1, 98-104.

[11] Tatum, E.T. (2018) Journal of Modern Physics, 9, 1867-1882. https://doi.org/10.4236/jmp.2018.910118

[12] Tatum, E.T. and Seshavatharam, U.V.S. (2018) Journal of Modern Physics, 9, 2008-2020. https://doi.org/10.4236/jmp.2018.910126

[13] Tatum, E.T. and Seshavatharam, U.V.S. (2018) Journal of Modern Physics, 9, 1404-1414. https://doi.org/10.4236/jmp.2018.97085

[14] Sapar, A. (2019) Proceedings of the Estonian Academy of Sciences, 68, 1-12. https://doi.org/10.3176/proc.2019.1.01

[15] Aghanim, N., et al. (2018) Planck 2018 Results VI. Cosmological Parameters. http://arXiv:1807.06209v1

[16] Macaulay, E., et al. (2018) First Cosmological Results Using Type Ia Supernovae from the Dark Energy Survey: Measurement of the Hubble Constant.

[17] Nielsen, J.T., et al. (2015) Scientific Reports, 6, Article No. 35596. https://doi.org/10.1038/srep35596

[18] Wei, J.-J., et al. (2015) Astronomical Journal, 149, 102. https://doi.org/10.1088/0004-6256/149/3/102

[19] Tutusaus, I., et al. (2017) Astronomy \& Astrophysics, 602, A73. https://doi.org/10.1051/0004-6361/201630289

[20] Dam, L.H., Heinesen, A. and Wiltshire, D.L. (2017) Monthly Notices of the Royal Astronomical Society, 472, 835-851. https://doi.org/10.1093/mnras/stx1858

[21] Perlmutter, S. (2016) Supernova Cosmology Project. Lawrence Berkeley National Laboratory, Berkeley. http://www.supernova.lbl.gov

[22] Suzuki, N., et al. (2011) The Hubble Space Telescope Cluster Supernovae Survey: V. Improving the Dark Energy Constraints above $\mathrm{Z}>1$ and Building an Early-Type-Hosted Supernova Sample.

[23] Tatum, E.T. and Seshavatharam, U.V.S. (2018) Journal of Modern Physics, 9, 1469-1483. https://doi.org/10.4236/jmp.2018.98091 